\title{
SAMUEL MULTIPLICITIES AND BROWDER SPECTRUM OF OPERATOR MATRICES
}

\author{
SHIFANG ZHANG AND JUNDE WU
}

\begin{abstract}
In this paper, we first point out that the necessity of Theorem 4 in [8] does not hold under the given condition and present a revised version with a little modification. Then we show that the definitions of some classes of semi-Fredholm operators, which use the language of algebra and first introduced by X. Fang in [8], are equivalent to that of some well-known operator classes. For example, the concept of shift-like semi-Fredholm operator on Hilbert space coincide with that of upper semi-Browder operator. For applications of Samuel multiplicities we characterize the sets of $\bigcap_{C \in B(K, H)} \sigma_{a b}\left(M_{C}\right), \bigcap_{C \in B(K, H)} \sigma_{s b}\left(M_{C}\right)$ and $\bigcap_{C \in B(K, H)} \sigma_{b}\left(M_{C}\right)$, respectively, where $M_{C}=\left(\begin{array}{cc}A & C \\ 0 & B\end{array}\right)$ denotes a 2-by-2 upper triangular operator matrix acting on the Hilbert space $H \oplus K$.

Mathematics subject classification (2010): Primary 47A10, Secondary 47A53.

Keywords and phrases: Samuel multiplicities, operator matrices, upper semi-Browder operator, upper semi-Browder spectrum, Browder operator, Browder spectrum.
\end{abstract}

\section{REFERENCES}

[1] X. H. CAO, Browder spectra for upper triangular operator matrices, J. Math. Anal. Appl. 342 (2008), $477-484$.

[2] X. L. Chen, S. F. ZhANG, H. J. ZHONG, On the filling in holes problem of operator matrices, Linear Algebra Appl. 430 (2009), 558-563.

[3] D. S. DJORDJEVIĆ, Perturbations of spectra of operator matrices, J. Operator Theory 48 (2002), 467-486.

[4] H. K. DU, J. PAN, Perturbation of spectrums of $2 \times 2$ operator matrices, Proc. Amer. Math. Soc. 121 (1994), 761-766.

[5] J. EschmeIER, Samuel multiplicity and Fredholm theory, Math. Ann. 339 (2007), 21-35.

[6] J. EschmeIER, On the Hilbert-Samuel multiplicy of Fredholm tuples, Indiana Univ. Math. J. 56 (2007), 1463-1477.

[7] J. ESCHMEIER, Samuel multiplicity for several commuting operators, J. Operator Theory 60 (2008), 399-414.

[8] X. FAnG, Samuel multiplicity and the structure of semi-Fredholm operators, Adv. Math. 1862 (2004), 411-437.

[9] X. FAnG, Hilbert polynomials and Arveson's curvature invariant, J. Funct. Anal. 198, 2 (2003), 445464.

[10] X. FANG, Invariant subspaces of the Dirichlet space and commutative algebra, J. Reine Angew. Math. 569 (2004), 189-211.

[11] X. FAnG, The Fredholm index of quotient Hilbert modules, Math. Res. Lett. 12 (2005), 911-920.

[12] X. FAnG, The Fredholm index of a pair of commuting operators, Geom. Funct. Anal. 16 (2006), $367-402$.

[13] J. K. HAN, H. Y. LEE, W. Y. LEE, Invertible completions of $2 \times 2$ upper triangular operator matrices, Proc. Amer. Math. Soc. 128 (1999), 119-123.

[14] I. S. HWANG, W. Y. LEE, The boundedness below of $2 \times 2$ upper triangular operator matrices, Integr. Equ. Oper. Theory 39 (2001), 267-276. 
[15] W. Y. LEE, Weyl's theorem for operator matrices, Integr. equ. oper. theory 32 (1998), 319-331.

[16] W. Y. LEE, Weyl spectra of operator matrices, Proc. Amer. Math. Soc. 129 (2000), 131-138.

[17] S. F. Zhang, H. J. Zhong, Q. F. JiAng, Drazin spectrum of operator matrices on the Banach space, Linear Algebra Appl. 429 (2008), 2067-2075.

[18] S. F. ZhANG, Z. Y. WU, H. J. ZHONG, Continuous spectrum, point spectrum and residual spectrum of operator matrices, Linear Algebra Appl. 433 (2010), 653-661.

[19] S. F. Zhang, H. J. Zhong, J. D. Wu, Spectra of Upper-triangular Operator Matrices, Acta Math. Sci. (in Chinese) 54 (2011), 41-60. 